\title{
Diálogo participativo - pensamiento crítico creativo en docentes de educación básica especial
}

\author{
Mg. Lina Silvia Chávez Quinteros \\ cquinterosls@ucvvirtual.edu.pe \\ https://orcid.org/0000-0001-6021-1991 \\ Mg. María Cecilia Delgado Orrillo \\ dorrillomc@ucvvirtual.edu.pe \\ https://orcid.org/0000-0003-1521-3175
}

Programa Académico de Doctorado en Educación

Escuela de postgrado Universidad César vallejo

Chiclayo - Perú

\section{RESUMEN}

La presente investigación tuvo como objetivo demostrar que el programa diálogo participativo desarrolló de forma óptima el pensamiento crítico y creativo en los docentes de educación básica especial de la provincia de Chiclayo. El tipo de investigación fue aplicada y de enfoque cuantitativo. La población correspondió a 60 docentes, trabajando con un grupo experimental y 01 grupo de control con 20 docentes en cada caso. Se administró un test con 30 ítems sobre el pensamiento crítico y creativo, debidamente validado. Los resultaron señalaron que la mayoría de docentes se ubicaron inicialmente, ambos grupos, en nivel bajo de desarrollo; así mismo participaron en el programa diálogo participativo para el desarrollo del pensamiento crítico y creativo, por medio de talleres especialmente dosificados concluyendo que, los docentes luego de la aplicación del programa de diálogo elevaron el desarrollo del pensamiento crítico y creativo.

Palabras clave: diálogo participativo; pensamiento crítico y creativo. 


\title{
Participatory dialogue - creative critical thinking in special basic education teachers
}

\begin{abstract}
The objective of this research was to demonstrate that the participatory dialogue program optimally developed critical and creative thinking in teachers of special basic education centers in the province of Chiclayo. The type of research was applied and with a quantitative approach. The population corresponded to 60 teachers, working with an experimental group and a control group with 20 teachers in each case. A duly validated test with 30 items on critical and creative thinking was administered, the results of which indicated that the majority of teachers in both groups were initially located at the low level of development; After participating in the participatory dialogue program for the development of critical and creative thinking, through specially dosed workshops they reached a high level; concluding that, after participating in the aforementioned program, teachers increased the development of critical and creative thinking
\end{abstract}

Keywords: participatory dialogue, critical and creative thinking

Artículo recibido: 30 noviembre. 2021 Aceptado para publicación: 29 diciembre 2021 Correspondencia: cquinterosls@ @cvvirtual.edu.pe Conflictos de Interés: Ninguna que declarar 


\section{INTRODUCCIÓN}

La educación es un proceso que con el transcurrir del tiempo ha pasado por constantes cambios, la labor del maestro en la actualidad es preparar a los estudiantes a adaptarse y generar cambios para el progreso, formando ciudadanos que generan nuevos aprendizajes, pensando de manera crítica y creativa dando igualdad de oportunidades para todos y todas.

En África Hughes (2020), considera la importancia del pensamiento crítico en el mundo, destaca que el Programa de la Escuela Primaria (PEP) y el Programa de los años intermedios (PAI) tienen como finalidad promover el pensamiento independiente. Entonces es fundamental tener en cuenta estas propuestas que benefician a los estudiantes con el desarrollo del pensamiento crítico.

Meller (2019) en Chile, plantea que la forma de enseñanza empleada en tiempos pasados no es útil para hoy, es necesario crear una sociedad del aprendizaje, desarrollando habilidades del siglo XXI, tales como el pensamiento socrático, promoviendo el cuestionamiento con planteamiento de interrogantes cuyas respuestas favorezcan la duda y se alcance la verdad.

Los docentes tienen inadecuadas formas de relacionar lo teórico, como señalan Moreno \& Velásquez (2017) indicando además que pocas veces utilizan estrategia alguna que favorezca desarrollar el pensamiento de los estudiantes, además estos no argumentan con métodos y estrategias problematizadoras. Siendo entonces necesario estimular la capacidad y habilidad del pensamiento del sujeto en todas sus direcciones.

En la provincia de Lambayeque, Hernández et al. (2020) desarrollaron una serie de conferencias sobre la situación educacional de las regiones Amazonas, Cajamarca y Lambayeque, resaltando que falta conocer lo que significa el pensamiento crítico y creativo (PCC), sus tipos y habilidades permiten realizar acciones de manera conjunta y responsable para tener una aceptable indagación referida a un contenido básico para solucionar problemas.

Estas apreciaciones del desarrollo del PCC en el mundo, permite conocer la importancia y preocupación por esta temática por lo que urge el conocimiento de estrategias específicas para aplicarlas con los maestros y estudiantes con la finalidad de elevar el 
nivel de desarrollo del PCC y con ello participar con más efectividad en el progreso de las sociedades.

El lugar donde se desarrolló la presente investigación fue la jurisdicción de la UGEL Chiclayo, con docentes de 09 Centros de Educación Básica Especial (CEBE), que de acuerdo al sistema NEXUS 2021 cuenta con 69 docentes.

Durante las asistencias técnicas realizadas a los directores de los CEBE se observó el problema de deficiencias en el desarrollo del PCC, reflejado en actitudes poco críticas y creativas ante los problemas que se generan a nivel de los estudiantes y de los CEBES en general con muchas limitaciones para hacer enjuiciamientos con fundamento teórico, reducida originalidad en la elaboración de trabajos educativos y el desarrollo de las sesiones de aprendizaje.

La problemática señalada tiene como causales desactualización en contenidos y técnicas para el desarrollo del PCC tanto para sí mismo como docentes como para los estudiantes de instituciones consideradas para la investigación, limitada participación en programas de diálogo participativo, dificultades para participar en cursos de actualización relacionados con el desarrollo del PCC.

Si este panorama problemático subsiste entonces los docentes de los CEBE no podrán profundizar el desarrollo del PCC, así mismos limitados para trabajar estos temas con sus estudiantes de acuerdo a sus características y el contexto correspondiente. Así mismo los docentes que no resuelvan la problemática indicada se verán imposibilitados de contribuir desde sus aulas con lo que plantea la OCDE (2020) y la ODS 20-30 sobre una educación de calidad sin distinción alguna, por lo tanto, con inclusión y equidad frente a los estudiantes de todos los niveles educativos.

La investigación tuvo como propósito diseñar y aplicar un programa de diálogo participativo orientado a fortalecer el desarrollo del PCC de los docentes que laboran en los centros de educación básica especial de la provincia de Chiclayo.

En relación a las conceptualizaciones encontradas respecto a la variable pensamiento crítico podemos decir que no hay una definición única, para Morancho \& Rodríguez (2020), el pensamiento crítico es un procedimiento que implica la capacidad de autorregular los procesos de aprendizaje eficaces que con el apoyo de la motivación y asociación de algunas destrezas, prácticas y sapiencias nos permiten construir una opinión planeada y reflexiva que nos conducirá al cumplimiento de la actividad o la solución de 
diversas soluciones problemáticas de forma competente y acertada. Es decir, pensar de forma crítica permite actuar de la forma más acertada ante las diversas situaciones que se nos pueda presentar.

Lo mismo ocurre con la variable creatividad, respecto a la variedad de conceptos, según Ibán et al. (2020), afirma que la creatividad es un proceso que agrupa y realiza una mixtura de diversos componentes y es en las aulas en donde se promueve el generar y explorar diversas opiniones En relación a la variable diálogo participativo, Córdoba (2020) señala que a través del diálogo respetuoso se ofrece la oportunidad de conversar y colocar en frases lo que emociona, además sirve para observar desde otro panorama nuestro actuar. Estos encuentros de diálogo participativo aparecen por decisión de cada grupo con la finalidad de realizar un buen trabajo con un mismo fin, dejando de lado identificaciones para competir, antes bien conocer a la persona por sus ideales, inclinaciones y sentimientos; el diálogo permite tener más conexión que surge desde el cariño por el campo educativo y por la persona.

Ante estos hechos, se formuló el problema de investigación, ¿En qué medida el programa de diálogo participativo repercute en el desarrollo del PCC en los docentes de los centros de educación básica especial de la provincia de Chiclayo, 2021?

El objetivo general es, demostrar que el programa de diálogo participativo influye en el fortalecimiento del desarrollo del pensamiento crítico y creativo en los docentes de los centros de educación básica especial de la provincia de Chiclayo, 2021. Entre los objetivos específicos se planteó, identificar el nivel de desarrollo del PCC de los docentes de los centros especiales a través de un pre-test, elaborar el programa diálogo participativo orientado al desarrollo del PCC, desarrollar el programa diálogo participativo a través de talleres con los docentes que participan en la investigación, evaluar el nivel PCC a través de un pos-test.

La investigación realizada es relevante por haber resuelto el problema del fortalecimiento del desarrollo del PCC de los docentes de los CEBES a través de un experimento específico aplicando el programa de diálogo participativo; cuyos resultados debidamente sistematizados se constituyen en el aporte teórico debido a que incrementa el conocimiento relacionado con el pensamiento crítico y creativo. 
Desde el punto de vista pedagógico y aplicativo la investigación muestra un aporte práctico por haber logrado que los docentes de los CEBES participen activamente en el programa de diálogo participativo especialmente diseñado.

Así mismo este estudio tiene beneficios metodológicos por haber utilizado técnicas, estrategias e instrumentos en la elaboración del programa diálogo participativo orientado al fortalecimiento del desarrollo del PCC, destacando con ello la precisión didáctica de los contenidos. Cabe señalar la repercusión social al beneficiar a los docentes participantes en la investigación y consecuentemente a los estudiantes con los cuales les toca laborar en las instituciones consideradas para la investigación.

La hipótesis que se planteó es que el programa diálogo participativo es un instrumento, que al ser aplicado repercute óptimamente en el desarrollo del pensamiento crítico y creativo en docentes de los centros de educación básica especial de Chiclayo

\section{ESTRATEGIAS METODOLÓGICAS}

\subsection{Tipo y diseño de investigación}

\subsubsection{Tipo de investigación: Aplicada.}

Permitió generar conocimientos y encontrar la solución a una problemática que se ha observado en torno al desarrollo del pensamiento crítico y creativo. Lozada, (2019) señala que la investigación aplicada tiene por finalidad producir el conocimiento que se obtiene de aplicar de forma inmediata y en un corto tiempo en una determinada comunidad. Es de enfoque metodológico cuantitativo por haber utilizado datos estadísticos con la finalidad de probar una hipótesis, se utilizó métodos estadísticos para extraer conclusiones y probar teorías (Hernández y Mendoza, 2018).

\subsubsection{Diseño investigación:}

El diseño de la presente investigación es experimental en el nivel cuasi-experimental porque se manipuló de forma deliberada la variable diálogo participativo y se apreció los efectos en el desarrollo del pensamiento crítico y creativo a través de un experimento de campo en una situación real. Hernández y Mendoza, (2018) refieren que los diseños cuasiexperimentales manejan de forma deliberada por lo mínimo una variable independiente con la finalidad de ver la consecuencia en una o más variables dependientes. El diagrama de investigación es el siguiente: 


$$
\begin{array}{cccc}
\text { GE } & : O_{1} & X & O_{2} \\
\text { GC }: & O_{3} & - & O_{4}
\end{array}
$$

\section{Donde:}

GE : Grupo Experimental

GC : Grupo de Control

$\mathrm{O}_{1} \quad$ : Test sobre pensamiento crítico y creativo aplicado a los docentes de los centros de educación básica especial Chiclayo que conformaron el grupo experimental, antes de la aplicación del programa diálogo participativo.

$\mathrm{X} \quad$ : Aplicación del programa diálogo participativo con los docentes que conforman el grupo de investigación.

$\mathrm{O}_{2} \quad$ : Test acerca del pensamiento crítico creativo aplicado a los docentes que participaron en la investigación, grupo experimental, después de la participación en el programa.

$\mathrm{O}_{3} \quad$ : Pre test acerca del pensamiento crítico creativo aplicado a los docentes del grupo de control.

$\mathrm{O}_{4} \quad$ : Post test sobre pensamiento crítico y creativo aplicado a los docentes que conformaron la muestra de estudio como grupo de control.

\subsection{Variables y Operacionalización}

\subsubsection{Variable 1 y/o independiente: diálogo participativo}

\subsubsection{Variable 2 y/o dependiente: pensamiento crítico y creativo}

\subsubsection{La operacionalización de las variables se encuentra ubicada en el anexo 01.}

\subsection{Población, muestra, muestreo}

\subsubsection{Población}

La población estuvo conformada por 60 docentes de los Centros de Educación Básica Especial de la provincia de Chiclayo. Se considera población a la unión de un grupo de asuntos, determinado, restringido y asequible, que constituirá el relativo para seleccionar la muestra que cumplirá con una sucesión de juicios ya establecidos (Arias et al. 2016). 


\section{Tabla 1}

Distribución del número de docentes de los Centros de Educación Básica Especial de la provincia de Chiclayo

\begin{tabular}{lcccc}
\multicolumn{1}{c}{ CEBES } & Distrito & Varones & Mujeres & Total \\
\hline "Venerable Francisco Camacho" & Tumán & 00 & 04 & 04 \\
"Santa Teresa de Jesús" & Oyotún & 00 & 03 & 03 \\
"Sagrado Corazón de Jesús" & Mocupe & 00 & 03 & 03 \\
"Santo Domingo" & Cayaltí & 01 & 02 & 03 \\
"Hogar Clínica San Juan de Dios" & Pimentel & 00 & 02 & 02 \\
"Niño Jesús de Praga" & Pimentel & 00 & 18 & 18 \\
"CERCILAM" & Chiclayo & 00 & 08 & 08 \\
"La Purísima" & Chiclayo & 00 & 09 & 09 \\
"La Victoria" & La Victoria & 00 & 10 & 10 \\
\hline Total : 09 CEBES & $\mathbf{0 7}$ distritos & $\mathbf{0 1}$ & $\mathbf{5 9}$ & $\mathbf{6 0}$ \\
\hline
\end{tabular}

Nota: Padrón NEXUS 2021.

La población estuvo conformada por docentes provenientes de 07 distritos de la provincia de Chiclayo donde funcionan centros de educación básica especial, conformado tanto por docentes nombrados y contratados con años de experiencia en la modalidad.

\subsubsection{Muestra}

La muestra fue determinada a criterio de la investigadora, 20 docentes para el grupo experimental y 20 para el grupo control utilizando la técnica probabilística del azar simple considerando los primeros 20 identificados que conformaron el grupo experimental y los restantes el grupo control. Hernández y Mendoza (2018), señala que es un subgrupo del total del que se va a recolectar los datos y que debe de representarla para que con ello se logre sistematizar el resultado.

\section{Tabla 2}

Distribución de la muestra de docentes de los centros de educación básica especial de la Provincia de Chiclayo

\begin{tabular}{|c|c|c|c|c|c|}
\hline \multirow{2}{*}{ Sección } & & \multicolumn{2}{|c|}{ Docentes } & \multirow{2}{*}{ Total } & \multirow{2}{*}{$\%$} \\
\hline & & Varones & Mujeres & & \\
\hline Grupo Experimental & Grupo A & 02 & 18 & 20 & 00 \\
\hline Sub total & & 02 & 18 & 20 & 50 \\
\hline Grupo Control & Grupo B & 00 & 20 & 20 & 00 \\
\hline Sub total & & 00 & 20 & 20 & 50 \\
\hline Total & & $\mathbf{0 2}$ & 38 & 40 & 100 \\
\hline
\end{tabular}

Nota: Padrón NEXUS 2021. 


\subsection{Técnicas e instrumentos de recolección de datos}

Se tuvo en cuenta la técnica de la observación, se realizó a través de un test compuesto por 30 ítems que estuvo dirigido a los docentes de los centros de educación básica especial de los distritos de Chiclayo que participaron en la investigación, el mismo que fue aplicado antes y después del experimento para recabar información referida a la variable dependiente pensamiento crítico y creativo (Anexo 03). Herrera (2020) señala que la observación es uno de los cimientos del conocimiento, es un argumento repetido y esencial, se refiere al recojo de datos previos a la investigación, es una acción que solicita de alguna confianza y cuidado, detenerse al recojo de informaciones y a las cosas forjadas que se presenta en lo que se conoce como el contexto.

La validez se realizó con la participación de 5 expertos a quienes se hizo llegar un expediente con los documentos pertinentes: carta de solicitud, matriz de consistencia, tabla de operacionalización, instrumentos, ficha de evaluación del instrumento dando conformidad a los instrumentos de las variables de estudio, recibiendo las recomendaciones correspondientes. El procesamiento de los datos se realizó a través de un indicador estadístico, utilizando para el coeficiente de validez la $\mathrm{V}$ de Aiken que permitió cuantificar la relevancia de los ítems referido a un dominio de contenido. El coeficiente que resultó tuvo el valor de 0.93 , lo que significó que el instrumento de recolección de datos tiene excelente validez (Anexo 04). Robles (2018), afirma que este coeficiente logra realizar la cuantificación de la validez de lo que contiene un instrumento y aplicarlo como un método que tiene lógica de validez cuando se cuenta con el dictamen de los expertos, el mismo que resulta de fácil cálculo y avala efectos sostenidos en técnicas estadísticos. La validez según Moral (2019), es comprobar si verdaderamente un instrumento evalúa lo que dice realizar.

La confiabilidad de los instrumentos se realizó a través de una prueba piloto a un grupo de 10 docentes que pertenecen a CEBES no considerados en el grupo experimental pero que cuentan con características similares. Para el tratamiento estadístico se empleó el programa SPSS y se aplicó el Alfa de Cronbrach buscando la fiabilidad en su consistencia interna, resultando 0.870 Arévalo y Padilla (2016) refieren que este programa logra la medición del nivel de confiabilidad de las pruebas, el entorno de su uso es amplio y permite medir la fiabilidad de las pruebas, las cuales mayormente tienen enfoque cuantitativo, requieren únicamente una sola aplicación del instrumento de medición, los 
valores que producen se encuentran entre 0 y 1 , no requiere la división de los ítems, ya que solo se aplica la medición y se computa el coeficiente. La confiabilidad según Moral (2019), es la capacidad de realizar una medición, pero con escaso margen de error.

Para la realización de esta investigación, se contó con la autorización del Director de la Unidad de la Unidad de Gestión Educativa Local Chiclayo, realizando la coordinación con los directores de los Centros de Educación Básica Especial para el apoyo de los docentes participantes, recolección de información, análisis documental, diseño de los instrumentos y la propuesta, validando los instrumentos e interpretando los datos.

Los datos recogidos con el instrumento de investigación fueron procesados utilizando el programa informático Excel y SPSSv26, derivando tablas, a través del cual se realizó el análisis correspondiente en base a las medidas de tendencia central y de dispersión obtenidas.

Se tuvo en cuenta el respeto a las personas que participaron en la investigación, se respetó la autoría de las fuentes bibliográficas revisadas, teniendo en cuenta lo establecido en el Código de ética sobre investigación (Universidad César Vallejo, 2020). Además, se consideraron las normas APA sétima edición para efecto de las citas y referencias bibliográficas,

\section{RESULTADOS}

Los resultados relacionados con el desarrollo del pensamiento crítico y creativo se resumen en la siguiente tabla considerando los grupos experimental y de control tanto a nivel de pre test y pos test.

\section{Tabla 03}

Nivel de logro de las variables pensamiento crítico y pensamiento creativo

\begin{tabular}{ccccccccc}
\hline & \multicolumn{4}{c}{$\begin{array}{c}\text { RESUMEN PENSAMIENTO CRÍTICO Y CREATIVO } \\
\text { Grupo Experimental }\end{array}$} & \multicolumn{3}{c}{$\begin{array}{c}\text { Grupo Control } \\
\text { Niveles de } \\
\text { logro }\end{array}$} & \multicolumn{2}{c}{$\begin{array}{c}\text { Pre test } \\
\mathbf{N}^{\circ}\end{array}$} & $\begin{array}{c}\text { Pos test } \\
\text { Pre test }\end{array}$ & $\mathbf{N}^{\circ}$ & $\%$ & $\mathbf{N}^{\circ}$ & $\%$ & $\mathbf{N}^{\circ}$ & $\begin{array}{c}\text { Pos test } \\
\text { \% }\end{array}$ \\
\hline Alto & 4 & 20.00 & 16 & 80.00 & 4 & 20.00 & 6 & 30.00 \\
Medio & 11 & 55.00 & 4 & 20.00 & 10 & 50.00 & 14 & 70.00 \\
Bajo & 5 & 25.00 & 0 & 0.00 & 6 & 30.00 & 0 & 0.00 \\
\hline TOTAL & $\mathbf{2 0}$ & $\mathbf{1 0 0 . 0 0}$ & $\mathbf{2 0}$ & $\mathbf{1 0 0 . 0 0}$ & $\mathbf{2 0}$ & $\mathbf{1 0 0 . 0 0}$ & $\mathbf{2 0}$ & $\mathbf{1 0 0 . 0 0}$ \\
\hline
\end{tabular}

Nota: Pre test y pos test aplicado al grupo experimental y grupo control 
En la tabla 03 se puede observar el consolidado de los niveles de logro de las variables de pensamiento crítico y pensamiento creativo, en lo que respecta al grupo experimental, en el pre test antes de la aplicación del programa diálogo participativo, se encuentran 05 docentes, es decir el $25 \%$ en el nivel bajo, medio 11 docentes que corresponde al $55 \%$ y en alto 04 docentes llegaron el $20 \%$, mientras que luego de aplicar el programa, en el pre test no existe el nivel bajo, en el nivel medio 04 docentes lograron el $20 \%$ y en un nivel alto 16 docentes alcanzaron el $80 \%$, por lo que se deduce que el programa diálogo participativo mejora de forma significativa el pensamiento crítico y creativo de los docentes de los centros de educación básica especial.

En lo que respecta al grupo control con la aplicación del pre test, en el nivel bajo se hallan 06 docentes equivalentes al $30 \%$, en el nivel medio 10 docentes que equivalen al $50 \%$ y en el nivel alto 04 docentes que corresponden al $20 \%$, con la aplicación del pos test no se cuenta con ningún estudiante en el nivel bajo, en el nivel medio 14 docentes, que equivalen al $70 \%$ y solo 06 docentes que corresponde al $30 \%$ se ubican en el nivel alto.

\section{DISCUSIÓN}

El mundo actual requiere formadores competentes, que generen en sus estudiantes habilidades para enfrentarse con éxito y puedan desenvolverse acertadamente ante las distintas situaciones que se puedan enfrentar cultivando siempre el pensamiento crítico y creativo.

En la presente investigación se ha tenido como objetivo demostrar que el programa de diálogo participativo influye en el fortalecimiento del desarrollo del pensamiento crítico y creativo en los docentes de los centros de educación básica especial de la provincia de Chiclayo, luego de su aplicación del programa a través de la estrategia diálogo participativo se tuvo como resultados que los docentes lograron situarse en el nivel alto en lo que respecta al pensamiento crítico y creativo, en comparación con el grupo control que no participó de los talleres que desarrolló el programa.

La propuesta pedagógica tuvo como fundamento teórico a Bandura con su teoría del aprendizaje social (1977), al respecto Sierra (2016) señala que los factores externos tienen igual importancia que los sucesos del ambiente, de los factores internos y que los comportamientos interactúan con el proceso de aprendizaje, además considera el enfoque de Competencias de Tobón (2006)quien propone una nueva forma de educación, basada en el espíritu humanista de la educación, con el pensamiento complejo como 
epistemología, con el individuo como cuerpo principal, para resolver los problemas prácticos que preocupan a la propia sociedad. Se considera además el modelo de pensamiento reflexivo, creativo y crítico de Villarini (2019) quien señala que el pensamiento crítico implica la participación de elementos que no están conscientes en el proceso de pensamiento, las conclusiones se pueden dar de forma contigua y se fabrican a partir de discernimientos y evocaciones y va más que solo producir el conocimiento.

El método que se ha empleado en la presente investigación es experimental en su nivel cuasi experimental, se trabajó con dos grupos, control y experimental, la selección de la muestra fue a criterio de la investigadora de acuerdo a cómo se recepcionaba el instrumento utilizado.

La propuesta que se diseñó fue la aplicación del programa diálogo participativo, a través de talleres donde se realizaron diversas temáticas sugeridas y captadas de los monitoreos que se realizó a los directivos y docentes, todos relacionados al PCC.

Los instrumentos de recolección de datos fueron debidamente validado por 05 expertos, quienes realizaron las observaciones requeridas dando las debidas sugerencias y recomendaciones para que el instrumento que se utilizó, test, sea adecuado y aplicable con la población que se aplicó.

Se realizó una prueba piloto con la participación de 20 docentes y determinar el nivel de pensamiento crítico y creativo en el que se encontraban y que permitieron loa planificación y ejecución de la propuesta diálogo participativo.

Las dificultades encontradas fueron las múltiples actividades de los docentes para el desarrollo de los talleres, aunados a la falta del manejo de la tecnología y a dificultades en las redes de internet, sobre todo de aquellos docentes que radican en lugares lejanos. De acuerdo a los resultados de las tablas podemos afirmar que el programa diálogo participativo mejora de forma significativa el pensamiento crítico y creativo de los docentes de los centros de educación básica especial. El resultado guarda relación con Suricachi, en Pasco (2018), en el que señala que el aprendizaje influye de manera positiva en la habilidad del pensamiento crítico y que se puede utilizar en diversas asignaturas ya que mejora las capacidades y el aprender, si se aplican diversos métodos, estrategias y/o programas de innovación se logra desarrollar las capacidades, competencias y conocimientos para mejorar habilidades, destrezas al resolver problemas, tomar decisiones, potenciando sus destrezas y sobre todo mejorar el pensamiento y creatividad. 


\section{CONCLUSIONES}

Se llegó a demostrar que la aplicación del programa diálogo participativo influye en el fortalecimiento del desarrollo del pensamiento crítico y creativo en los docentes de los centros de educación básica especial de la provincia de Chiclayo. Los docentes de los centros de educación básica especial de la UGEL Chiclayo que participaron del programa diálogo participativo, tanto del grupo de control y experimental inicialmente se ubicaron en el nivel bajo del desarrollo del pensamiento crítico y creativo. La investigación realizada permitió elaborar un programa de diálogo participativo considerando los resultados, nivel bajo, obtenidos luego de la aplicación del pre test sobre el pensamiento crítico y creativo a ambos grupos de investigación. Para el desarrollo del pensamiento crítico y creativo se ejecutaron talleres didácticamente elaborados con los docentes que formaron parte del grupo experimental en la presente investigación. Los docentes de los CEBES UGEL Chiclayo, grupo experimental, luego de participar en el programa diálogo participativo alcanzaron un nivel alto en el desarrollo del pensamiento crítico y creativo, lo que no sucedió con los docentes del grupo de control. 


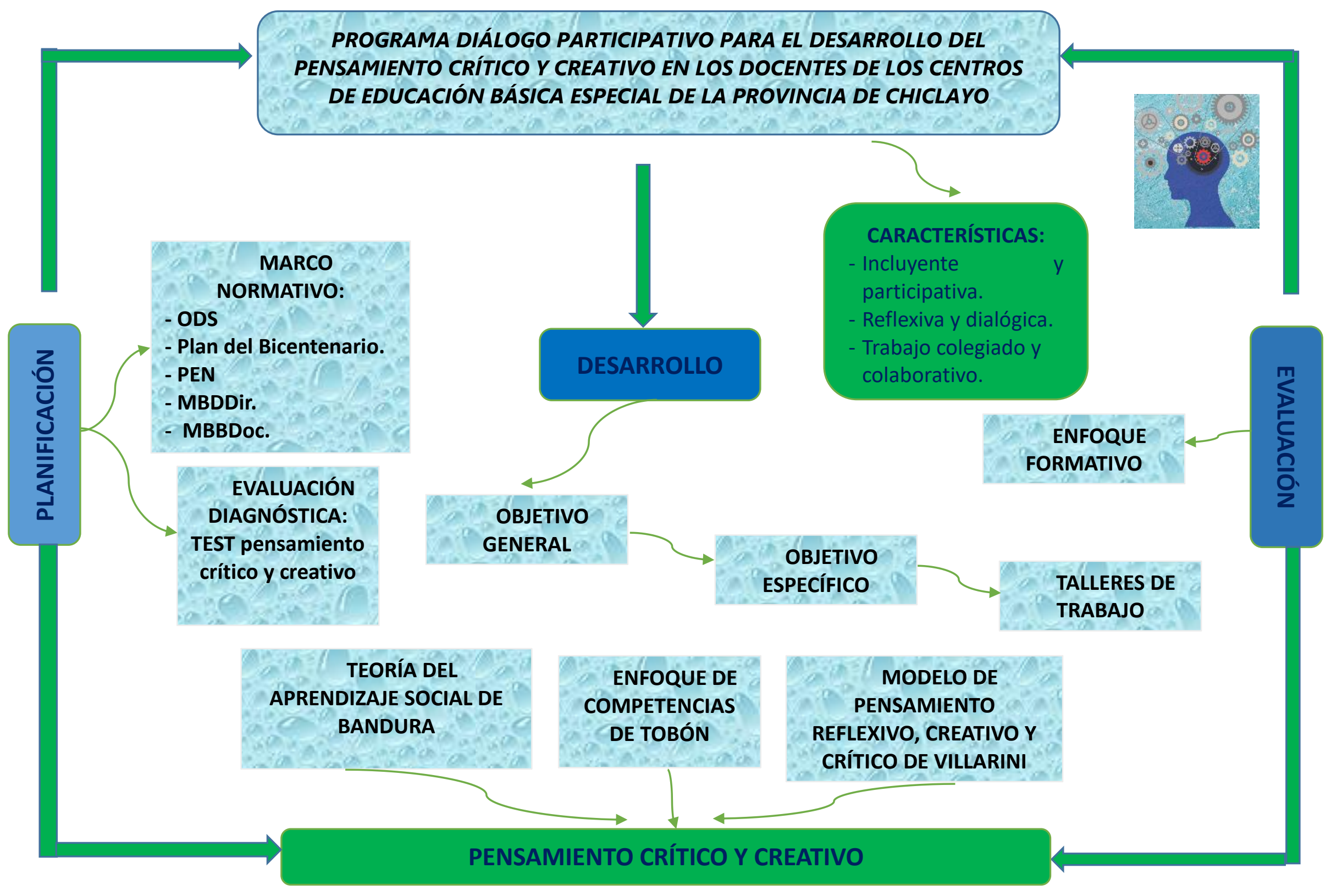

Ciencia Latina Revista Científica Multidisciplinar, Ciudad de México, México.

ISN 2707-2207 / ISSN 2707-2215 (en línea), enero- febrero, 2022, Volumen 6, Número 1. https://doi.org/10.37811/cl_rcm.v6i1.1546 p861 


\section{REFERENCIAS BIBLIOGRAFICAS}

Arévalo, D., Padilla, C. Medición de la Confiabilidad del Aprendizaje del Programa RStudio Mediante Alfa de Cronbach. Revista Politécnica. https://revistapolitecnica.epn.edu.ec/images/revista/volumen37/tomo1/Medicion_ de_la_Confiabilidad_del_Aprendizaje_del_Programa_RStudio_Mediante_Alfa_d e_Cronbach.pdf

Cordoba, W. (2020). Espacios de diálogo para la transición hacia el modelo social de atención a las personas con discapacidad: Una mirada desde la investigación acción participativa. Revista Electrónica Educare. https://www.scielo.sa.cr/scielo.php?pid=S1409$\underline{42582020000100394 \& \text { script }=\text { sci_arttext }}$

Haviz, M., Maris, I., (2020). Measuring mathematics and science teachers' perception on thinking and acting in 21st-century learning. Journal for the Education of Gifted Young Scientists, 8(4), 1319-1328. https://doi.org/10.17478/jegys.747395

Herrera, P.(2020). Reflexiones sobre la Observación. https://wiki.ead.pucv.cl/images/d/db/T9_-

_Reflexiones_sobre_la_Observaci\%C3\%B3n_\%281\%29.pdf

Hernández, R., Fernández, C., Baptista, M. (2014). Metodología de la Investigación. https://dialnet.unirioja.es/descarga/articulo/6163749.pdf

Hernández, R., Mendoza C. (2018). Metodología de la Investigación. Las rutas cuantitativa, cualitativa y mixta. file:///C:/Users/usuario/Downloads/2018\%20HERNANDEZ\%20SAMPIERI.pdf

Ibán, R., Marcos, S., López, V., Teresa, M., González, D., \& Phillips-silver, J. (2020). Promoting children' $s$ creative thinking through reading and writing in a cooperative learning classroom. Thinking Skills and Creativity, 36(June 2019), 100663. https://doi.org/10.1016/j.tsc.2020.100663

Lozada J. (2014). Investigación Aplicada: Definición, Propiedad Intelectual e Industria. https://dialnet.unirioja.es/descarga/articulo/6163749.pdf

Meller, P (2019) PSU y Simce. Chilenos deberán evaluar pensamiento crítico y creatividad. Chile. Revista el economista América. https://www.eleconomista.es/autor/elEconomistaAmericacom-Chile 
Moral, J., (2019). Revisión de los criterios para validez convergente estimada a través de la Varianza Media http://www.scielo.org.co/scielo.php?script=sci_arttext\&pid=S1900$\underline{23862019000200025 \# B 15}$

Morancho, M., Rodríguez J.(2020) Pensamiento Crítico: conceptualización y relevancia en el seno de la educación superior. Revista De La Educación Superior, 49(194), 925. http://resu.anuies.mx/ojs/index.php/resu/article/view/1121

Moreno y Velásquez (2016). Estrategia Didáctica para Desarrollar el Pensamiento Crítico REICE. Revista Iberoamericana sobre Calidad, Eficacia y Cambio en Educación, 2017, 15(2), 53-73. https://doi.org/10.15366/reice2017.15.2.003

OCDE. (2019). El trabajo de la OCDE sobre educación y competencias. Documento de la OCDE. https://www.oecd.org/education/El-trabajo-de-la-ocde-sobre-educaciony-competencias.pdf

ODS (2020). Objetivos de Desarrollo sostenible 20-30. La Agenda para el Desarrollo Sostenible. https://www.un.org/sustainabledevelopment/es/development-agenda/ Posligua J., Chenche W., Vallejo B. (2017). Incidencia de las actividades lúdicas en el desarrollo del pensamiento. Revista Científica Dominio de las ciencias. Vol. 3, núm. $\quad 3, \quad$ junio, $\quad$ 2017, $1020-1052$ https://dominiodelasciencias.com/ojs/index.php/es/index

Resolución de Consejo Universitario N 0262-2020/UCV

file:///C:/Users/usuario/Downloads/6\%20RCUN\%C2\%B00262-2020UCV\%20Apr.C\%C3\%B3d.Etica\%20Investigaci\%C3\%B3n.pdf

Robles, B. (2018). Indice de validez de contenido: Coeficiente V de Aiken. Pueblo Continente. http://200.62.226.189/PuebloContinente/article/view/991/914

Sierra, C. (2016). Teoría Social del Aprendizaje de Albert Bandura (Archivo PDF] https://www.academia.edu/30920256/_Teor\%C3\%ADa_del_Aprendizaje_Social de_Albert_Bandura_Cecilia_Sierra_E_Psicolog\%C3\%ADa_de_la_Infancia_Ju lio_2016_UNAG_pdf

Surichaqui, W. (2018). Aprendizaje basado en Problemas y habilidades de Pensamiento Crítico en la escuela profesional de Secundaria de la Universidad Daniel Alcides Carrión de la Universidad Daniel Alcides Carrión de la Región Pasco: 2016 [Tesis Doctoral. Universidad de Extremadura]. 
https://repositorio.usmp.edu.pe/bitstream/handle/20.500.12727/4527/surichaqui hwi.pdf? sequence $=1 \&$ is Allowed $=\mathrm{y}$

Tobón S. (2006). Aspectos básicos de la formación basada en competencias https://www.uv.mx/rmipe/files/2019/07/Aspectos-basicos-de-la-formacionbasada-en-competencias.pdf

Villarini A. (2019). Un modelo del pensamiento reflexivo, creativo y crítico como competencia humana general. Revista Internacional Magisterio. https://www.magisterio.com.co/articulo/un-modelo-del-pensamiento-reflexivocreativo-y-critico-como-competencia-humana-general 